\title{
Identifying friction stir welding process parameters through coupled numerical and experimental analysis
}

\author{
Xingguo Zhou, Wenke Pan*, Donald MacKenzie \\ University of Strathclyde, 75 Montrose Street, Glasgow G1 1XJ, UK
}

Keywords:

Friction stir welding

Genetic algorithm

Automatic parameter identification

Numerical modeling

Python

\begin{abstract}
A B S T R A C T
Friction Stir Welding (FSW) is a complex thermal-mechanical process. Numerical models have been used to calculate the thermal field, distortion and residual stress in welded components but some modeling parameters such as film coefficient and thermal radiation of the work pieces may be technically difficult and/or expensive to measure experimentally. Therefore, it is important to establish a systematic procedure to identify FSW process parameters. In this paper, a simplified finite element model for analysis of a FSW thermal progress is proposed in which two parameters, tool heat input rate and heat loss through the backing plate, are identified as parameters for optimization through application of a generic algorithm. A genetic algorithm is used to evaluate the two thermal parameters. By comparing the FEM numerical results with experimental results, the FSW process thermal parameters have been successfully identified. This automatic parameters characterization procedure could be used for the FSW process optimization.
\end{abstract}

(c) 2013 Published by Elsevier Ltd.

\section{Introduction}

Invented in 1991 by The Welding Institute, Friction Stir Welding is an advanced welding technology for joining materials in solid state. Compared to conventional fusion welding, FSW can achieve good weld mechanical properties and it is especially suitable for welding high strength aluminum. FSW is not only a user friendly process but also an environmentally friendly process because it doesn't emit toxic gases nor UV rays which are harmful to human health. Furthermore, low residual stress and distortion is expected, making the technique attractive for welding large sheet material.

FSW was initially developed for joining low-weldability aluminium alloys, but has since been used to join a variety of materials in a range of industry sectors. Fig. 1 shows a schematic drawing of a FSW tool and workpiece. The whole FSW process involves four stages: plunge, dwell, traverse and retraction. A cylindrical pin with a larger shoulder rotates and gradually plunges to a certain depth into the material and then dwells for certain time to let the workpiece temperature reach the optimal welding temperature, then traverses with constant speed along the weld line to the finish point and finally retracts from the workpiece.

The temperature history plays a very important role in final grain size and distribution and dissolution of precipitates and

\footnotetext{
* Corresponding author. Tel.: +4401415482036.

E-mail address: wenke.pan@strath.ac.uk (W. Pan).
}

consequently affects the mechanical properties of the weld. By using the Rosenthal equation, Gould [1] investigated the temperature distribution of FSW workpiece with the assumption that heat was solely generated by the work done by the friction force between the workpiece and tool shoulder. Vilaca et al. [2] also used the Rosenthal equation to deduce the analytical solution for thermal distribution formulation by placing the point heat source at the middle plane along the thickness direction of the workpiece and considered the heat generated from not only the translation but also the rotation movement of the welding tool.

Apart from the simplified analytical thermal solution of FSW, different types of numerical thermal modeling methods were also studied by researchers. Chao [3] et al. initially presented a 3-D thermal model including heat generation from the shoulder. Later, they used an inverse method to obtain the thermal parameters with the analysis of the separated models of workpiece and tool [4]. In Colegrove's model [5], heat generated from shearing of the material and friction on the vertical and threaded surface of the pin were studied. By correlating the heat input with the experimentally measured torque and considering different types of contact conditions including sliding, sticking and partial sliding/ sticking, a 3-D thermal model was analyzed by Khandkar et al. [6] A detailed review on the simplified FSW thermal model can be found in Ref. [7].

However, as FSW is a complicated thermal-mechanical process, some modeling parameters such as film coefficient and thermal radiation of the work pieces may be technically difficult and/or 


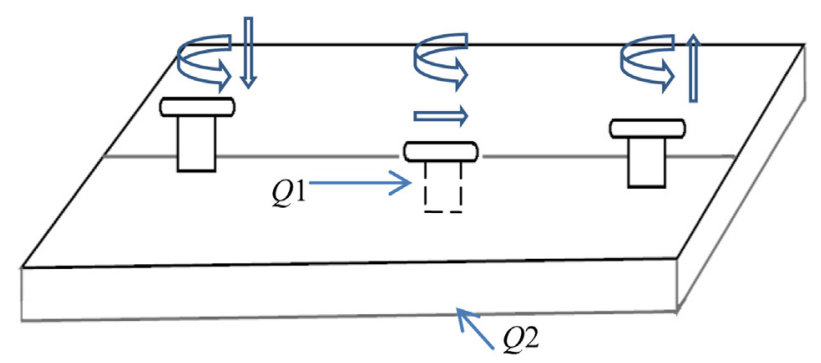

Fig. 1. Schematic drawing of FSW tool and workpiece: Q1 presents heat input, Q2 presents heat dissipation to support plate.

expensive to measure experimentally. Previous research work on numerical FSW thermal modeling mainly focused on trial and error methods to obtain these thermal parameters. This may be very time consuming or difficult to obtain optimal values. Therefore, it is important to establish a systematic procedure to identify FSW process parameters.

In this paper, an automatic FSW process for thermal parameters characterization is presented. For validation of the proposed method, experiment results from Ref. [4] are used for comparison. In the following section, a brief introduction of how ABAQUS Python commands are used to manage a high performance cluster by allocating finite element jobs to different nodes to perform parallel simulation is given. An investigation of a basic finite element thermal model, which was used for the thermal parameters identification in the later simulation, is presented. Section 4 is about the genetic algorithm for optimization of thermal parameters, while in Section 5 , the results are given. The final section is the conclusions and further work.

\section{ABAQUS python scripts for parallel simulation}

The identification process for FSW parameters includes: (1) to build a trial FEM thermal model with guess values for the parameters to be identified (2) to employ a genetic algorithm (GA) method to obtain the optimal values for these parameters (3) to use high performance clusters for the simulation of FEM model with the parameters updated by GA procedure. Due to the large amount of FEM modeling involved, a systematic procedure has to be developed. The proper use of ABAQUS python scripts could provide an ideal way to deal with this problem. Before the start of the ABAQUS python procedure, a basic FEM thermal model will be established (see Section 3). The FEM results will be compared with that of the theoretical results and the model with good results will be kept for the later simulation. Fig. 2 shows the flow chart used for thermal parameters optimization. Firstly, the basic ABAQUS model file is called and the heat input and surface film coefficient user subroutines are prepared. Secondly, the GA module is called to modify the thermal parameters in user subroutines. Each GA generation consists of 20 seeds, therefore, 20 FEM models are prepared.

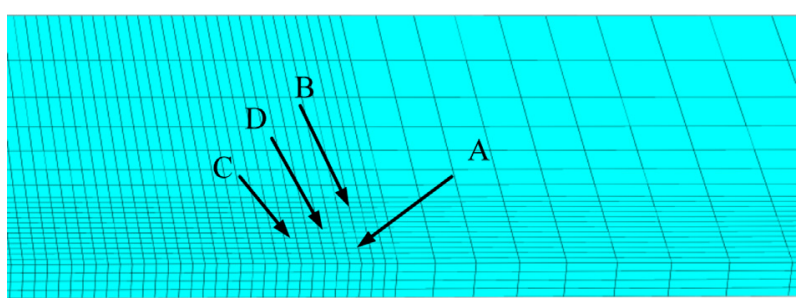

Fig. 3. FEM mesh of simplified FSW 3D ABAQUS thermal model. A point heat source is applied at the central point along the weld line.

Thirdly, 20 different ABAQUS jobs are allocated and submitted to different HPC nodes. Fourthly, after these jobs have finished, the ABAQUS post-processing program is called to extract the temperature history data at the points of interest for all the models. By comparing the calculated results with that of experimental results, the whole procedure will stop if the convergence criterion is satisfied or the total iteration number reached. Otherwise, go to second step and repeat the second step to fourth step until the defined criterions satisfied.

\section{FSW finite element thermal model}

To validate the proposed FSW process thermal parameters identification method, the FSW experiment results from Ref. [4] are used for comparison. In ref. 4, two aluminum plates of length of $610 \mathrm{~mm}$, width $102 \mathrm{~mm}$ and thickness $8.1 \mathrm{~mm}$ are friction stir welded. The case with tool traverse speed of $2.36 \mathrm{~mm} / \mathrm{s}$ is studied in this paper. The thermal history data at the point (named as Point $\mathrm{C}$ for convenience) $5 \mathrm{~mm}$ from the weld line on top surface is used for thermal parameter characterization, while the temperature variation with time at another point (named as point $\mathrm{D}$ ) on middle plane of the plate with same distance as point $C$ from the central line is employed for validation.

To obtain the optimized values for the FSW thermal parameters, large numbers of FEM models has to be analyzed. Because only the thermal parameters are to be identified, a basic FEM model will be analyzed and then this model will serve as a model for other simulations. The only change for other models is the modification of $Q 1$ and Q2 (see Fig. 1) and their values can be written into the models through ABAQUS user subroutines.

\subsection{Basic thermal model}

Fig. 3 shows a simplified FSW 3D transient thermal model with a continuous point heat source applied at point $A$, i.e. the centre point along the welding line. As two identical aluminum plates are to be welded, a half model is analyzed. The ABAQUS 3D 8-node brick element is employed and the model contains 19,215 nodes and 14,560 elements. The material thermal conductivity and specific heat are taken from Ref. [4]. Assuming no heat dissipation from the

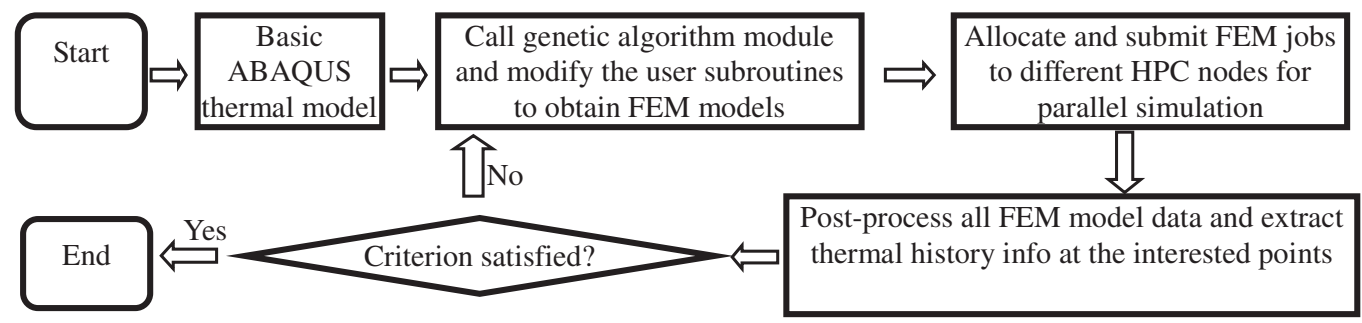

Fig. 2. ABAQUS python procedure for parameters optimization. 


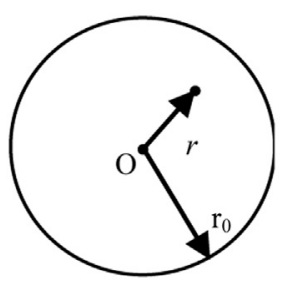

$$
q(r)=\frac{3 Q_{1} r}{2 \pi r_{0}^{3}}
$$

Fig. 4. Heat input rate distribution under the tool shoulder.

workpiece, the numerical results can be compared with analytical solution where a point heat source is applied to an infinite plate. The analytical temperature formulation for any point at time $t$ is given as follows [8].

$T(R, t)=\frac{q}{2 \pi \lambda R}\left[1-\Phi\left(\frac{R}{\sqrt{4 a t}}\right)\right]$

where the heat rate $q$, conductivity $\lambda$, specific heat $c$ and density $\rho$ are $1000 \mathrm{w}, 120 \mathrm{w} / \mathrm{m}^{2}, 1000 \mathrm{w} / \mathrm{m}^{3}$ and $2700 \mathrm{~kg} / \mathrm{m}^{3}$ respectively. While $a=\lambda /\left(c^{*} \rho\right)$ and $\Phi(u)$ is the probability integral function. $R$ is the distance from the heat source, $t$ is time in second.

Fig. 5 is the comparison of the temperature history between the numerical and theoretical results at point $B(10 \mathrm{~mm}$ from point $A$ along the line perpendicular to the weld line). From this figure, it can be seen that the numerical results are in good agreement with that of the analytical results within $1.1 \mathrm{~s}$, but with the time increases, the error becomes bigger. This error is mainly because the heat can be accumulated in the numerical model with small thickness, while for the theoretical model the heat can be transferred to the far area. The error is not caused by the mesh and integration step time, therefore this model serves as a basic model for the later simulation.

\subsection{FSW FEM model for thermal parameters characterization}

The thermal model for FSW is based on the basic thermal model. Instead of a continuous point heat source, a moving heat source with a constant total heat input rate $q(r)$ labeled as $Q 1$ in Equ. 2, to be identified, from the tool shoulder is assumed, but the heat input rate for each point under the shoulder will be proportional to the distance $(r)$ from the point to the centre of the shoulder, as shown in Fig. 4, where $r_{0}$ is the radius of the tool shoulder.

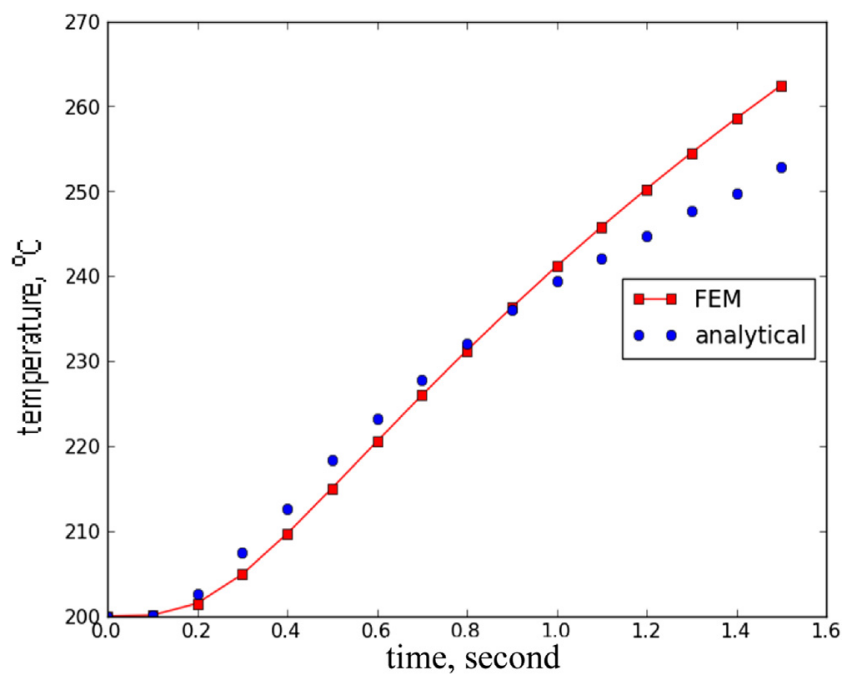

Fig. 5. Comparison of temperature result between FEM and analytical model.
Table 1

Conductivity property of workpiece [4].

\begin{tabular}{llllllll}
\hline Conductivity & 90 & 100 & 110 & 120 & 130 & 140 & 150 \\
Temperature & 0 & 50 & 100 & 150 & 200 & 250 & 300 \\
\hline
\end{tabular}

Table 2

Specific heat of workpiece [4].

\begin{tabular}{lllll}
\hline Specific heat & 900 & 1000 & 1077 & 1100 \\
Temperature & 0 & 150 & 250 & 300 \\
\hline
\end{tabular}

The heat transferred from the workpiece to the support plate is considered through a thermal film coefficient, defined as Q2 with uniform distribution, to be identified. The advantage of introducing Q2 is that there is no need to include the support plate into the plate. Hence, the total CPU time for the simulation will be reduced.

$q(r)=\frac{3 Q_{1} r}{2 \pi r_{0}^{3}}$

The heat dissipated from the side and the top surface of the workpiece to air is considered through the use of thermal film coefficient with a value of $30 \mathrm{w} / \mathrm{m}^{2}$ in the FE model. Due to symmetry, no heat exchange occurs on the middle plane along the weld line. The initial temperature for the whole workpiece is assumed to be $25{ }^{\circ} \mathrm{C}$. Material properties, including specific heat and conductivity, are assumed to vary with temperature, and are listed in Table 1 and Table 2 respectively [4].

\section{Genetic algorithm for thermal parameters characterization}

Based on the natural rule of fitter creatures having more chance to survive, GA is one type of optimization method especially suitable for parallel simulation. In this paper, the optimal values for $Q 1$ and $Q 2$ are to be identified. In using GA, $Q 1$ and $Q 2$ will be represented by an integral (with 8 bits of 0 and 1 ) with the range of $0-255$. The real values of $Q 1$ and $Q 2$ can be calculated from the integral using the formulation shown in Fig. 6 with a given range for $Q 1$ and $Q 2$, vice versa. In this case, searching ranges for $Q 1$ and $Q 2$ are assumed as $[1000 \mathrm{w}, 2000 \mathrm{w}]$ and $\left[100 \mathrm{w} / \mathrm{m}^{2} /{ }^{\circ} \mathrm{C}, 500 \mathrm{w} / \mathrm{m}^{2} /{ }^{\circ} \mathrm{C}\right]$ respectively.

The flow chart of GA process is shown in Fig. 7. Initially, 20 sets of chromosomes corresponding to $Q 1$ and $Q 2$ are randomly generated. Then the real values of $Q 1$ and $Q 2$ are calculated. Follow this, 20 FEM thermal model jobs are prepared and submitted to the HPC. After the solutions are complete, the temperature history data is extracted at the points of interest such as point $C$ and $D$ defined in Section 3.1.

This will follow the calculation of the value of the objective function $\delta$ for each seed, defined as:

$\delta=\sqrt{\frac{1}{n} \sum_{i=1}^{n}\left(\frac{T_{\text {cal }}^{i}-T_{\text {exp }}^{i}}{T_{\text {exp }}^{i}}\right)^{2}}$

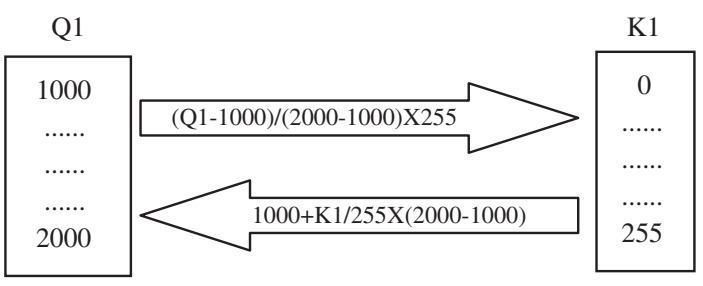

Fig. 6. Real value of $Q 1$ can be transferred to integral from 0 to 255 , vice versa. 


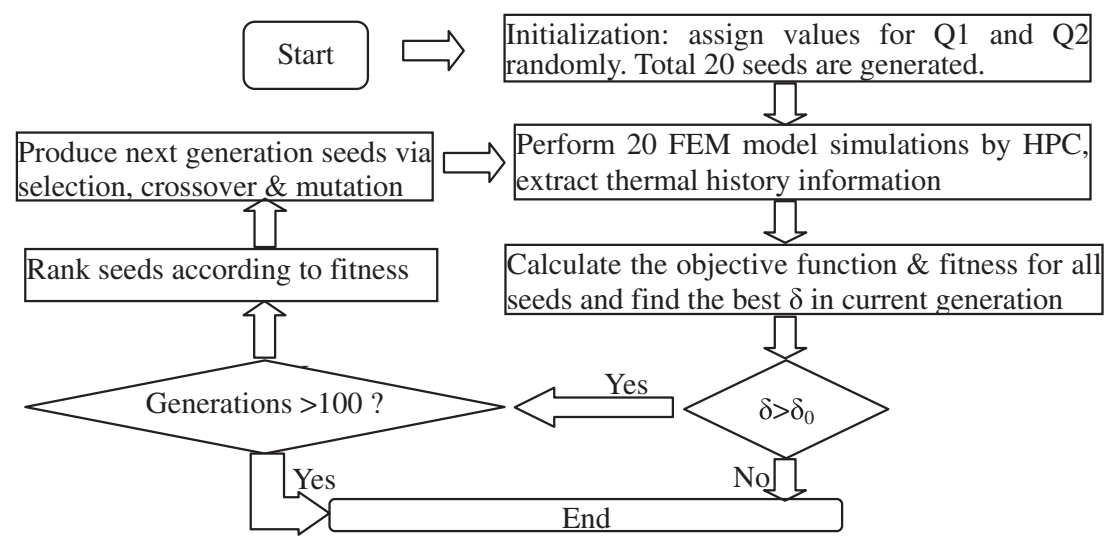

Fig. 7. GA flow chart for identification of FSW process thermal parameters.

where $T_{\exp }^{i}$ and $T_{\text {cal }}^{i}$ are the experimental and calculated temperature for the $i$ th seed respectively. ' $n$ ' represents the total points extracted from the temperature vs time curves. The fitness for each seed is defined as $f=1 / \delta$, and the largest value of fitness in one generation is selected as the fitness of the generation. Finally, if the value of $\delta$ is smaller than a given small value or the total number of generation is larger than 100 , the GA process stops. Otherwise, new off-spring for next generation will be created through selection, cross-over and mutation. These steps are repeated until the criterion is satisfied.

\section{Numerical results}

Based on the GA flowchart shown in Fig. 7 and the ABAQUS Python procedure shown in Fig. 2, both the GA module and ABAQUS Python codes are developed. The temperature history [4] at point $C$ (see Section 3) are divided into 20 segments as shown in Fig. 7, therefore 21 experiment data points are used for calculating the GA objective function value.

The data points were chosen purposely after $100 \mathrm{~s}$ of welding because the temperature is not sensitive to the welding time at an earlier stage. After GA evolution to the 35th generation, the average

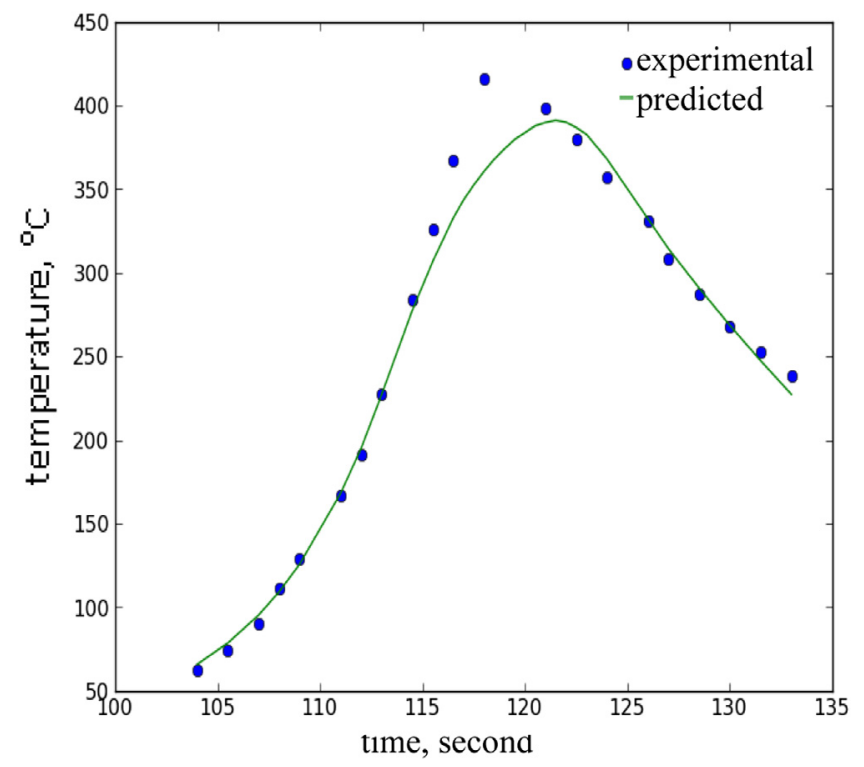

Fig. 8. Comparison of experimental [4] \& predicted temperature at point C.

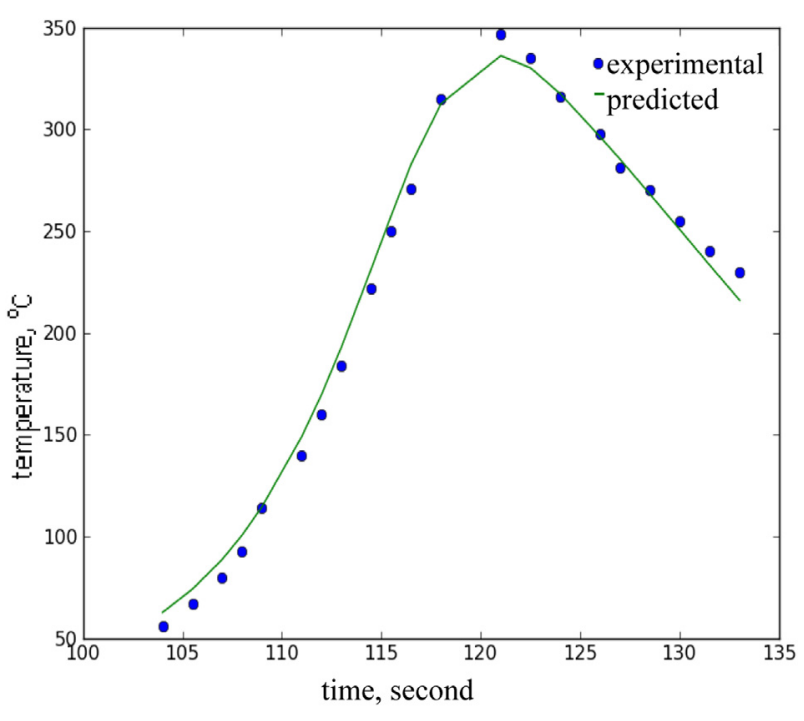

Fig. 9. Comparison of experimental [4] \& predicted temperature at point D.

relative error between the experimental and numerical results is smaller than 5\%. The values for the thermal parameters Q1 and Q2 obtained are $1714 \mathrm{~W}$ and $462 \mathrm{~W} / \mathrm{m}^{2}$. By using these identified parameters and performing ABAQUS simulation again, the comparison of

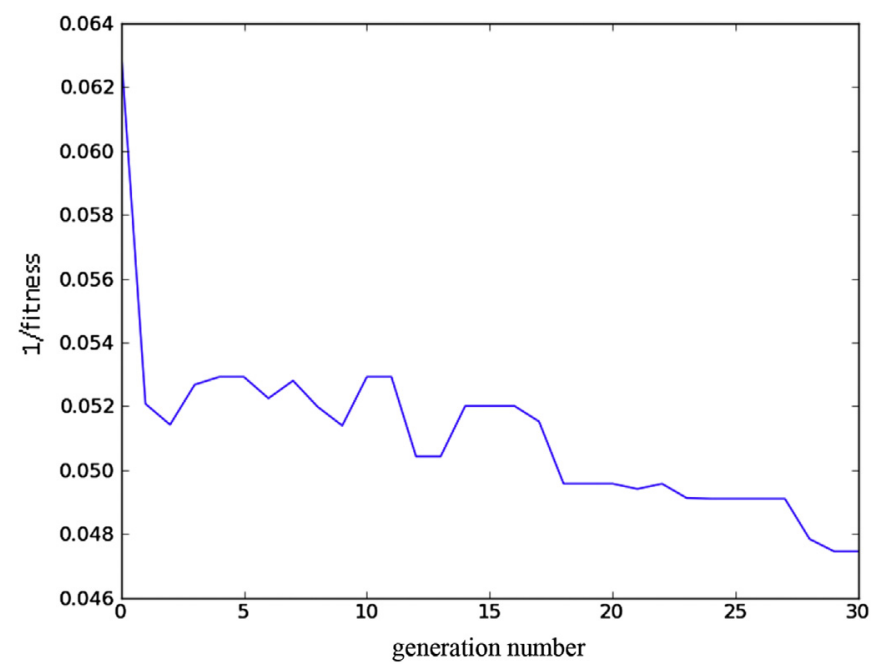

Fig. 10. Fitness evolution history. 
experimental and numerical results at point $C$ is shown in Fig. 7. This shows good agreement between the results of the two methods. The thermal history at another point $\mathrm{D}$ is also predicted and depicted in Fig. 9. A similar conclusion can be made as that from Fig. 8. This comparison shows that the proposed numerical model is reliable and accurate because the measured temperature data at point $\mathrm{D}$ has not been included into the thermal parameters characterization procedure. In Fig. 10 the relative error between the predicted and experimental results is plotted against GA iteration number.

\section{Conclusions and future work}

Taking advantage of high performance cluster parallel computing and the commercial Finite Element software ABAQUS python codes, the Finite Element Method was coupled with a genetic algorithm optimization to obtain the best value for the thermal input (heat from a moving heat source simulating friction stir welding) and thermal film coefficient (between the workpiece and support plate). By using the predicted parameters from one set of experiment results, the temperature distribution at other points are predicted and found to be in good agreement with the experimental results. The heat input predicted is also similar to that obtained in Refs. [4], in which a general inverse method is used. The optimization procedure presented in this paper performs the parameter identification automatically and could be extended to include the complex features of the welding tool. As the temperature history plays a very important part of the microstructure in welded zones, this systematic procedure could be used for FSW process optimization. Future work will include the thermal mechanical coupling analysis to predict the residual stress and distortion.

\section{Acknowledgments}

The authors would like to thank Scottish Overseas Research Student Awards Scheme and University of Strathclyde for financial supporting the research.

\section{References}

[1] Gould J. Heat flow model for friction stir welding of aluminum alloys. J Mater Process Manuf Sci 1998;7:185-94.

[2] Vilaca P, Quintino L, Santos JF. iSTIR-analystical thermal model for friction stir welding. J Mater Process Tech 2005;169(3):452-65.

[3] Chao YJ, Qi X. Thermal and Thermo-mechanical modelling of friction stir welding of aluminum alloy 6061-T6 plates. J Mater Process Manuf Sci 1998;7: 215-33.

[4] Chao YJ, Qi X, Tang W. Heat Transfer in friction stir welding-experimental and numerical Studies. J Manuf Sci Eng 2003;125:138-45.

[5] Colegrove P. 3-Dimentional Flow and Thermal Modelling of the Friction Stir Welding Process. Proceedings of the 2nd International Symposium on Friction Stir Welding, Sweden, August 2000.

[6] Khandkar MZH, Khan JA, Reynolds AP. A Thermal Model of the Friction Stir Welding Process', Proceedings of IMECE2002, ASME International Mechanical Engineering Congress \& Exposition, New Orleans, Louisiana, November, 2002.

[7] Li HJ. Coupled thermo-mechanical modelling of friction stir welding. PhD thesis. UK: University of Strathclyde; 2008.

[8] Zhang WY. Heat transfer theory for welding. Mechanical Industry Press, ISBN 711101667X; 1989. p. 27-8. 